\title{
FERMI/LARGE AREA TELESCOPE DISCOVERY OF GAMMA-RAY EMISSION FROM A RELATIVISTIC JET IN THE NARROW-LINE QUASAR PMN J0948+0022
}

A. A. Abdo ${ }^{1,49}$, M. AckermanN ${ }^{2}$, M. Ajello ${ }^{2}$, M. Axelsson ${ }^{3,4}$, L. Baldini $^{5}$, J. Ballet $^{6}$, G. Barbiellini $^{7,8}$, D. Bastieri $^{9,10}$, M. Battelino ${ }^{3,11}$, B. M. Baughman ${ }^{12}$, K. Bechtol ${ }^{2}$, R. Bellazzini ${ }^{5}$, E. D. Bloom ${ }^{2}$, E. Bonamente ${ }^{13,14}$, A. W. Borgland ${ }^{2}$, J. Bregeon ${ }^{5}$, A. Brez ${ }^{5}$, M. Brigida ${ }^{15,16}$, P. Bruel ${ }^{17}$, G. A. Caliandro ${ }^{15,16}$, R. A. Cameron ${ }^{2}$, P. A. Caraveo ${ }^{18}$, J. $^{2}$ M. Casandian ${ }^{6}$, E. Cavazzuti ${ }^{19}$, C. Cecchi ${ }^{13,14}$, A. Chekhtman ${ }^{1,20}$, C. C. Cheung ${ }^{21}$, J. Chiang ${ }^{2}$, S. Ciprini ${ }^{13,14}$, R. Claus ${ }^{2}$, J. Cohen-Tanugi ${ }^{22}$, W. Collmar ${ }^{23}$, J. Conrad ${ }^{3,11,24,50}$, L. Costamante ${ }^{2}$, C. D. Dermer ${ }^{1}$, A. De Angelis ${ }^{25}$, F. De Palma $^{15,16}$, S. W. Digel ${ }^{2}$, E. do Couto e Silva ${ }^{2}$, P. S. Drell ${ }^{2}$, R. Dubois ${ }^{2}$, D. Dumora ${ }^{26,27}$, C. FARnier ${ }^{22}$, C. FAVuzZi ${ }^{15,16}$, W. B. Focke ${ }^{2}$, L. Foschini ${ }^{28,51}$, M. Frailis ${ }^{25}$, L. Fuhrmann ${ }^{29}$, Y. Fukazawa ${ }^{30}$, S. Funk ${ }^{2}$, P. Fusco ${ }^{15}, 16$, F. Gargano ${ }^{16}$, N. Gehrels ${ }^{21,31}$, S. Germani ${ }^{13,14}$, B. Giebels ${ }^{17}$, N. Giglietto ${ }^{15,16}$, F. Giordano ${ }^{15,16}$, M. Giroletti ${ }^{32}$, T. GlanZMAN ${ }^{2}$, I. A. Grenier ${ }^{6}$, M.-H. Grondin ${ }^{26,27}$, J. E. Grove ${ }^{1}$, L. Guillemot ${ }^{26,27}$, S. Guiriec ${ }^{33}$, Y. Hanabata ${ }^{30}$, A. K. Harding ${ }^{21}$, R. C. Hartman ${ }^{21}$, M. HAyAShida ${ }^{2}$, E. Hays ${ }^{21}$, R. E. Hughes ${ }^{12}$, G. Jóhannesson ${ }^{2}$, A. S. Johnson ${ }^{2}$, R. P. Johnson ${ }^{34}$, W. N. Johnson ${ }^{1}$, T. KAMAE ${ }^{2}$, H. Katagiri ${ }^{30}$, J. KataOKA ${ }^{35}$, M. KerR ${ }^{36}$, J. KNÖDlseder ${ }^{37}$, F. Kuehn ${ }^{12}$, M. Kuss ${ }^{5}$, J. Lande ${ }^{2}$, L. LAtronico ${ }^{5}$,

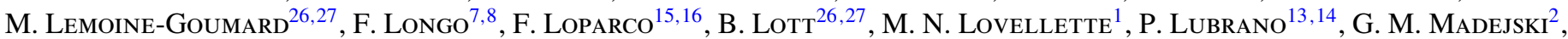
A. Makeev ${ }^{1,20}$, W. MaX-Moerbeck ${ }^{38}$, M. N. Mazziotta ${ }^{16}$, W. McConville ${ }^{21,31}$, J. E. McEnery ${ }^{21}$, C. Meurer $^{3,24}$, P. $^{2}$ F. Michelson ${ }^{2}$, W. Mitthumsiri ${ }^{2}$, T. Mizuno ${ }^{30}$, C. Monte ${ }^{15}, 16$, M. E. Monzani ${ }^{2}$, A. Morselli ${ }^{39}$, I. V. Moskalenko $^{2}$, S. Murgia ${ }^{2}$, P. L. Nolan ${ }^{2}$, J. P. Norris ${ }^{40}$, E. Nuss ${ }^{22}$, T. Ohsugi ${ }^{30}$, N. OModei ${ }^{5}$, E. Orlando ${ }^{23}$, J. F. Ormes ${ }^{40}$, D. Paneque ${ }^{2}$, J. H. Panetta ${ }^{2}$, D. Parent ${ }^{26,27}$, V. Pavlidou ${ }^{38}$, T. J. Pearson ${ }^{38}$, M. Pepe ${ }^{13,14}$, M. Pesce-Rollins ${ }^{5}$, F. Piron $^{22}$, T. A. Porter $^{34}$, S. RAINÒ ${ }^{15,16}$, R. RANDO ${ }^{9,10}$, M. RAZZANO ${ }^{5}$, A. READHEAD ${ }^{38}$, A. REIMER ${ }^{2}$, O. REIMER ${ }^{2}$, T. REPOSEUR ${ }^{26,27}$, J. L. RICHARDS $^{38}$, S. Ritz ${ }^{21}$, A. Y. Rodriguez ${ }^{41}$, R. W. Romani ${ }^{2}$, F. Ryde ${ }^{3,11}$, H. F.-W. SADrozinski ${ }^{34}$, R. SAMBrUnA ${ }^{21}$, D. SANCHEZ ${ }^{17}$, A. Sander ${ }^{12}$, P. M. Saz Parkinson ${ }^{34}$, J. D. Scargle ${ }^{48}$, T. L. Schalk ${ }^{34}$, C. Sgrò ${ }^{5}$, D. A. Smith ${ }^{26,27}$, G. Spandre $^{5}$, P. Spinelli ${ }^{15}, 16$, J.-L. Starck ${ }^{6}$, M. Stevenson ${ }^{38}$, M. S. Strickman ${ }^{1}$, D. J. Suson ${ }^{42}$, G. Tagliaferri ${ }^{28}$, H. TaKahashi $^{30}$, T. TANAKA ${ }^{2}$, J. G. ThaYeR ${ }^{2}$, D. J. Thompson ${ }^{21}$, L. Tibaldo $^{9,10}$, O. Tibolla $^{43}$, D. F. Torres ${ }^{41,44}$, G. Tosti $^{13,14}$,

A. Tramacere ${ }^{2,45}$, Y. Uchiyama ${ }^{2}$, T. L. Usher ${ }^{2}$, N. Vilchez ${ }^{37}$, V. Vitale ${ }^{39,46}$, A. P. Waite ${ }^{2}$, B. L. Winer ${ }^{12}$, K. S. Wood ${ }^{1}$, T. YLINEN ${ }^{3,11,47}$, J. A. Zensus ${ }^{29}$, M. ZIEGLER ${ }^{34}$

(The Fermi/LAT Collaboration)

AND

G. Ghisellini $^{28}$, L. Maraschi ${ }^{28}$, F. TAvecchio ${ }^{28}$, E. Angelakis ${ }^{29}$

${ }^{1}$ Space Science Division, Naval Research Laboratory, Washington, DC 20375, USA

${ }^{2}$ W. W. Hansen Experimental Physics Laboratory, Kavli Institute for Particle Astrophysics and Cosmology, Department of Physics and SLAC National Accelerator Laboratory, Stanford University, Stanford, CA 94305, USA

${ }^{3}$ The Oskar Klein Centre for Cosmo Particle Physics, AlbaNova, SE-106 91 Stockholm, Sweden

${ }^{4}$ Department of Astronomy, Stockholm University, SE-106 91 Stockholm, Sweden

5 Istituto Nazionale di Fisica Nucleare, Sezione di Pisa, I-56127 Pisa, Italy

${ }^{6}$ Laboratoire AIM, CEA-IRFU/CNRS/Université Paris Diderot, Service d'Astrophysique, CEA Saclay, F-91191 Gif sur Yvette, France

${ }^{7}$ Istituto Nazionale di Fisica Nucleare, Sezione di Trieste, I-34127 Trieste, Italy

${ }^{8}$ Dipartimento di Fisica, Università di Trieste, I-34127 Trieste, Italy

${ }^{9}$ Istituto Nazionale di Fisica Nucleare, Sezione di Padova, I-35131 Padova, Italy

${ }^{10}$ Dipartimento di Fisica "G. Galilei," Università di Padova, I-35131 Padova, Italy

${ }^{11}$ Department of Physics, Royal Institute of Technology (KTH), AlbaNova, SE-106 91 Stockholm, Sweden

12 Department of Physics, Center for Cosmology and Astro-Particle Physics, The Ohio State University, Columbus, OH 43210, USA

${ }^{13}$ Istituto Nazionale di Fisica Nucleare, Sezione di Perugia, I-06123 Perugia, Italy

${ }^{14}$ Dipartimento di Fisica, Università degli Studi di Perugia, I-06123 Perugia, Italy

${ }^{15}$ Dipartimento di Fisica "M. Merlin" dell'Università e del Politecnico di Bari, I-70126 Bari, Italy

${ }^{16}$ Istituto Nazionale di Fisica Nucleare, Sezione di Bari, I-70126 Bari, Italy

${ }^{17}$ Laboratoire Leprince-Ringuet, École polytechnique, CNRS/IN2P3, F-91128 Palaiseau, France

18 INAF-Istituto di Astrofisica Spaziale e Fisica Cosmica, I-20133 Milano, Italy

${ }^{19}$ Agenzia Spaziale Italiana (ASI) Science Data Center, I-00044 Frascati (Roma), Italy

${ }^{20}$ George Mason University, Fairfax, VA 22030, USA

${ }^{21}$ NASA Goddard Space Flight Center, Greenbelt, MD 20771, USA

${ }^{22}$ Laboratoire de Physique Théorique et Astroparticules, Université Montpellier 2, CNRS/IN2P3, F-34095 Montpellier, France

${ }_{23}^{3}$ Max-Planck Institut für extraterrestrische Physik, D-85748 Garching, Germany

${ }^{24}$ Department of Physics, Stockholm University, AlbaNova, SE-106 91 Stockholm, Sweden

${ }^{25}$ Dipartimento di Fisica, Università di Udine and Istituto Nazionale di Fisica Nucleare, Sezione di Trieste, Gruppo Collegato di Udine, I-33100 Udine, Italy

${ }^{26}$ CNRS/IN2P3, Centre d'Études Nucléaires Bordeaux Gradignan, UMR 5797, F-33175 Gradignan, France

${ }^{27}$ Université de Bordeaux, Centre d'Études Nucléaires Bordeaux Gradignan, UMR 5797, Gradignan, France

${ }^{28}$ INAF Osservatorio Astronomico di Brera, I-23807 Merate, Italy; luigi.foschini @ brera.inaf.it

${ }^{29}$ Max-Planck-Institut für Radioastronomie, Auf dem Hügel 69, D-53121 Bonn, Germany

${ }^{30}$ Department of Physical Sciences, Hiroshima University, Higashi-Hiroshima, Hiroshima 739-8526, Japan

${ }^{31}$ University of Maryland, College Park, MD 20742, USA

${ }^{32}$ INAF Istituto di Radioastronomia, I-40129 Bologna, Italy

${ }^{33}$ University of Alabama in Huntsville, Huntsville, AL 35899, USA

${ }^{34}$ Santa Cruz Institute for Particle Physics, Department of Physics and Department of Astronomy and Astrophysics, University of California at Santa Cruz, Santa Cruz, CA 95064, USA 


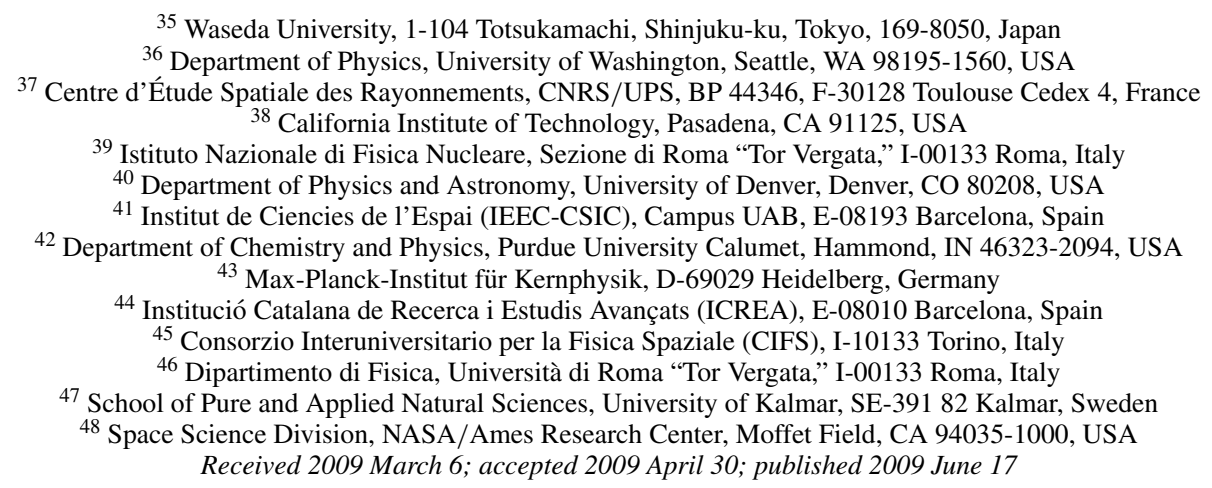

\section{ABSTRACT}

We report the discovery by the Large Area Telescope (LAT) onboard the Fermi Gamma-Ray Space Telescope of high-energy $\gamma$-ray emission from the peculiar quasar PMN J0948+0022 $(z=0.5846)$. The optical spectrum of this object exhibits rather narrow $\mathrm{H} \beta\left(\operatorname{FWHM}(\mathrm{H} \beta) \sim 1500 \mathrm{~km} \mathrm{~s}^{-1}\right)$, weak forbidden lines, and is therefore classified as a narrow-line type I quasar. This class of objects is thought to have relatively small black hole mass and to accrete at a high Eddington ratio. The radio loudness and variability of the compact radio core indicate the presence of a relativistic jet. Quasi-simultaneous radio/optical/X-ray and $\gamma$-ray observations are presented. Both radio and $\gamma$-ray emissions (observed over five months) are strongly variable. The simultaneous optical and X-ray data from Swift show a blue continuum attributed to the accretion disk and a hard X-ray spectrum attributed to the jet. The resulting broadband spectral energy distribution (SED) and, in particular, the $\gamma$-ray spectrum measured by Fermi are similar to those of more powerful Flat-Spectrum Radio Quasars (FSRQs). A comparison of the radio and $\gamma$-ray characteristics of PMN J0948+0022 with the other blazars detected by LAT shows that this source has a relatively low radio and $\gamma$-ray power with respect to other FSRQs. The physical parameters obtained from modeling the SED also fall at the low power end of the FSRQ parameter region discussed in Celotti \& Ghisellini. We suggest that the similarity of the SED of PMN J0948+0022 to that of more massive and more powerful quasars can be understood in a scenario in which the SED properties depend on the Eddington ratio rather than on the absolute power.

Key words: galaxies: active - gamma rays: observations - quasars: individual (PMN J0948+0022)

Online-only material: color figure

\section{INTRODUCTION}

It is now widely recognized that strong radio sources associated with active galactic nuclei (AGNs) must be powered by collimated relativistic energy flows (Rees 1966). The bulk Lorentz factors $(\Gamma)$ of these flows may be different in different systems and at different distances from the active nucleus. If a blob of plasma moving at relativistic speed is observed at small angles to the jet axis $(\theta \leqslant 1 / \Gamma)$, the observed radiation is amplified and the timescales shortened due to relativistic effects. Such systems are generically called blazars (Blandford \& Rees 1978). Blazars have often been classified in subcategories: Flat-Spectrum Radio Quasars (FSRQs), characterized by strong and broad optical emission lines, and BL Lac objects, when no emission lines are apparent above the optical/UV continuum (e.g., Urry \& Padovani 1995).

Remarkably, the Compton Gamma-Ray Observatory (CGRO) together with the first generation of Cerenkov telescopes discovered that the spectral energy distribution (SED) of a number of the brightest blazars extend to the $\gamma$-ray range, showing two broad components: the first one, covering radio to soft X-rays, is thought to be due to the synchrotron emission from relativistic electrons, while the second one, covering the hard $\mathrm{X}-/ \gamma$-ray

\footnotetext{
${ }^{49}$ National Research Council Research Associate.

${ }^{50}$ Royal Swedish Academy of Sciences Research Fellow, funded by a grant from the K. A. Wallenberg Foundation.

${ }^{51}$ Author to whom any correspondence should be addressed.
}

band, is generally attributed to inverse-Compton (IC) emission. The seed photons for the IC process can originate from the synchrotron radiation itself (synchrotron self-Compton (SSC); e.g., Ghisellini et al. 1985) or from an external source, such as the accretion disk, the broad-line region, or a dusty torus (external Compton (EC); e.g., Dermer et al. 1992; Sikora et al. 1994; Błażejowski et al. 2000).

Compiling and averaging the SED of the brightest blazars, Fossati et al. (1998) found an interesting trend-the blazar sequence - whereby for sources with low bolometric luminosity both components peak at high frequencies $(\mathrm{UV} /$ soft X-rays for synchrotron and $\mathrm{TeV}$ for IC-high-frequency peaked BL Lacs (HBL)), while, for increasing luminosities, both peaks shift to lower frequencies (low-frequency peaked BL Lacs (LBL) and FSRQ).

Ghisellini et al. (1998) proposed to explain the sequence in terms of correlation between the random Lorentz factor of electrons emitting at the peaks of the $\operatorname{SED}\left(\gamma_{\text {peak }}\right)$ and the global energy density $(U)$ in the comoving frame. HBL have low $U$ and high $\gamma_{\text {peak }}$, while FSRQ have high $U$ and low $\gamma_{\text {peak }}$. The sequence can also be interpreted in an evolutionary frame (Böttcher \& Dermer 2002; Cavaliere \& D'Elia 2002).

It is important to stress that the selection of objects with which the sequence was constructed was admittedly biased by the available samples, within which only a limited number of objects had $\gamma$-ray data (Maraschi \& Tavecchio 2001). In fact challenges have been raised to the validity of the sequence (for a review, see Padovani 2007 and references therein), which 
however could be overcome (Maraschi et al. 2008; Ghisellini \& Tavecchio 2008). Today, the ongoing Fermi mission is expected to provide a deeper and unbiased survey of the whole $\gamma$-ray sky, compared to that available during the CGRO/EGRET era, yielding possible surprises, as we will show in the present work.

Although the origin of relativistic jets is presently still not understood, there is increasing evidence that the properties of jets are related to the properties of the accretion flow which feeds the central black hole. In the case of stellar mass black holes, the observed phenomenology points to an association of jet launching with accretion "modes" characterized by different spectral and timing properties (Fender \& Belloni 2004). In the extragalactic domain, the separation of radio sources into two broad classes (FRI and FRII), as well as the properties of their respective "beamed" representatives, BL Lacs and FSRQs, can be basically understood within a scenario based on the accretion mode: in the first class accretion onto the central black hole is sub-critical (in Eddington units) leading to radiatively inefficient accretion flows and relatively weak jets, while in the second one the accretion rate is near critical, giving rise to bright disks and powerful jets (Ghisellini \& Celotti 2001; Maraschi 2001; Maraschi \& Tavecchio 2003; however, see also Blandford \& Levinson 1995).

In this respect, the case of radio-loud narrow-line Seyfert 1 (NLS1) active nuclei has received increasing attention. NLS1 are characterized by an optical spectrum with narrow permitted lines $\operatorname{FWHM}(\mathrm{H} \beta)<2000 \mathrm{~km} \mathrm{~s}^{-1}$, the ratio of $[\mathrm{O}$ III $] \lambda 5007$ to $\mathrm{H} \beta$ smaller than 3 , and a bump due to Fe II (see, e.g., Pogge 2000 for a review). They also exhibit prominent soft $\mathrm{X}$-ray excesses. These properties point to very high (near Eddington) accretion rates and relatively low masses $\left(10^{6}\right.$ $10^{8} M_{\odot}$; Boroson 2002; however, see Decarli et al. 2008; Marconi et al. 2008). Only a small percentage of NLS1 are radio-loud $\left(R L=\left(S_{v=4.85 \mathrm{GHz}} / S_{v=440 \mathrm{~nm}}\right)>10\right)$ or very radioloud $(R L>100)(7 \%$ and $2.5 \%$, respectively). Their flat radio spectra suggest that several of them could host relativistic jets; in fact, very long baseline interferometry (VLBI) variability indicates extremely high brightness temperatures and in some cases superluminal expansion has been observed (Komossa et al. 2006; Doi et al. 2006).

Recently, Yuan et al. (2008) studied a complete sample of radio-loud NLS1 selected from the Sloan Digital Sky Survey (SDSS) sample. They found that a large fraction of those for which X-ray data exist show broadband spectra similar to those of HBL, with peaks close to the UV band. The study of simultaneous optical/UV/X-ray data of a sample of radioloud NLS1 revealed that these sources often display a hard $\mathrm{X}$-ray component, especially in bright optical and UV states, thus supporting the possible contribution of a relativistic jet, in some cases similar to FSRQs (Foschini et al. 2009).

This class of sources is thus of extreme interest for extending the studies of the properties of relativistic jets to different mass and power scales. While it is clear that the most radioloud NLS1 should host relativistic jets, the properties of such jets at high energy are essentially unknown. Observations of a few selected sources in the $\mathrm{TeV}$ energy range with the Whipple and HESS Cerenkov telescopes were performed, but yielded only upper limits (Falcone et al. 2004; Aharonian et al. 2008). A detection at high energy ( $\mathrm{GeV}$ or $\mathrm{TeV} \gamma$-rays) is essential to complete the knowledge about the SED, allowing one to constrain the IC parameters and discuss analogies and differences with previously known blazars.
Here we present the first detection, by the Fermi/Large Array Telescope (LAT), of $\gamma$-rays from one of these radioloud NLS1, the quasar PMN J0948+0022 $(z=0.5846)$. This makes it possible to build the first whole SED from radio to $\gamma$-rays of radio-loud NLS1, to constrain the IC emission, and to evaluate the role of this new type of source in the framework of the blazar sequence and evolution. The paper is organized as follows: after a short presentation of the source in Section 2, the analysis of Fermi/LAT, Swift, Effelsberg, and Owens Valley Radio Observatory (OVRO) data is presented in Section 3; Section 4 deals with the SED and the model selected to fit to the broadband spectrum, while the discussion and conclusions are given in Section 5. Throughout this work, we adopted a $\Lambda \mathrm{CDM}$ cosmology from the most recent Wilkinson Microwave Anisotropy Probe (WMAP) results, which give the following values for the cosmological parameters: $h=0.71, \Omega_{m}=0.27$, $\Omega_{\Lambda}=0.73$, and with the Hubble-Lemaitre constant $H_{0}=100 \mathrm{~h}$ $\mathrm{km} \mathrm{s}^{-1} \mathrm{Mpc}^{-1}$ (Komatsu et al. 2009).

\section{THE SOURCE PMN J0948+0022}

First identified in the MIT-Green Bank radio survey at $5 \mathrm{GHz}$ (Bennett et al. 1986), this quasar is part of the sample drawn from the SDSS and Faint Images of the Radio Sky at Twenty $\mathrm{cm}$ (FIRST) in a systematic search for radio-loud NLS1 objects (Zhou et al. 2003, 2006; Komossa et al. 2006; Yuan et al. 2008). The flux ratio $[\mathrm{O} \mathrm{III}] / \mathrm{H} \beta=0.1<3$ (according to the most recent measurements by Zhou et al. (2006)) indicates that the Balmer lines are indeed originated from the usual broad-line region, meaning that this is not a Type II AGN (Zhou et al. 2003). There is no obscured broad-line region, as supported by the absence of any additional absorption in the optical (see the analyses by Zhou et al. 2003, 2006) and X-ray spectra (no additional $N_{\mathrm{H}}$ is required in the fit of $S w i f t / \mathrm{X}$-ray Telescope (XRT) data in the present work; see Section 3.2). A reanalysis of SDSS data by Zhou et al. (2006) succeeded in separating the $\mathrm{H} \beta$ into a broad and narrow component (see also RodríguezArdila et al. 2000), where the "broad" component has FWHM $=1432 \pm 87 \mathrm{~km} \mathrm{~s}^{-1}$. Therefore, PMN J0948+0022 has a broadand a narrow-line region, but the FWHM of the "broad" lines is small, less than $2000 \mathrm{~km} \mathrm{~s}^{-1}$.

Its strong and variable radio emission $(R L>1000)$, together with a flat and inverted radio spectrum $\left(\alpha_{r}=-0.24\right.$, with $\alpha$ defined as $S_{v} \propto v^{-\alpha}$ ), makes PMN J0948+0022 one of the few undoubtedly radio-loud narrow-line Seyfert 1 quasars. In addition, VLBI observations at different epochs revealed high brightness temperatures and significant flux density variations, requiring a Doppler factor $\delta>2.5$ and a viewing angle $<22^{\circ}$ (Doi et al. 2006). Now, a $\gamma$-ray detection is reported in the first list of bright $\gamma$-ray sources (significance $>10 \sigma$ ) detected by Fermi/LAT (Abdo et al. 2009b) and, specifically, in the List of Bright AGN Sources (LBAS; Abdo et al. 2009c).

\section{DATA ANALYSIS}

\subsection{Fermi/LAT}

PMN J0948+0022 appears in the LBAS derived from the first three months of the Fermi/LAT all-sky survey. It is associated (93\% confidence level) with the source 0FGL J0948.3+0019 (Abdo et al. 2009b, 2009c). In order to improve the positional accuracy for this source and hence increase the confidence level of the association, we added two more months of data to the three months of the LBAS, thus providing five months of data (from 2008 August through December). 


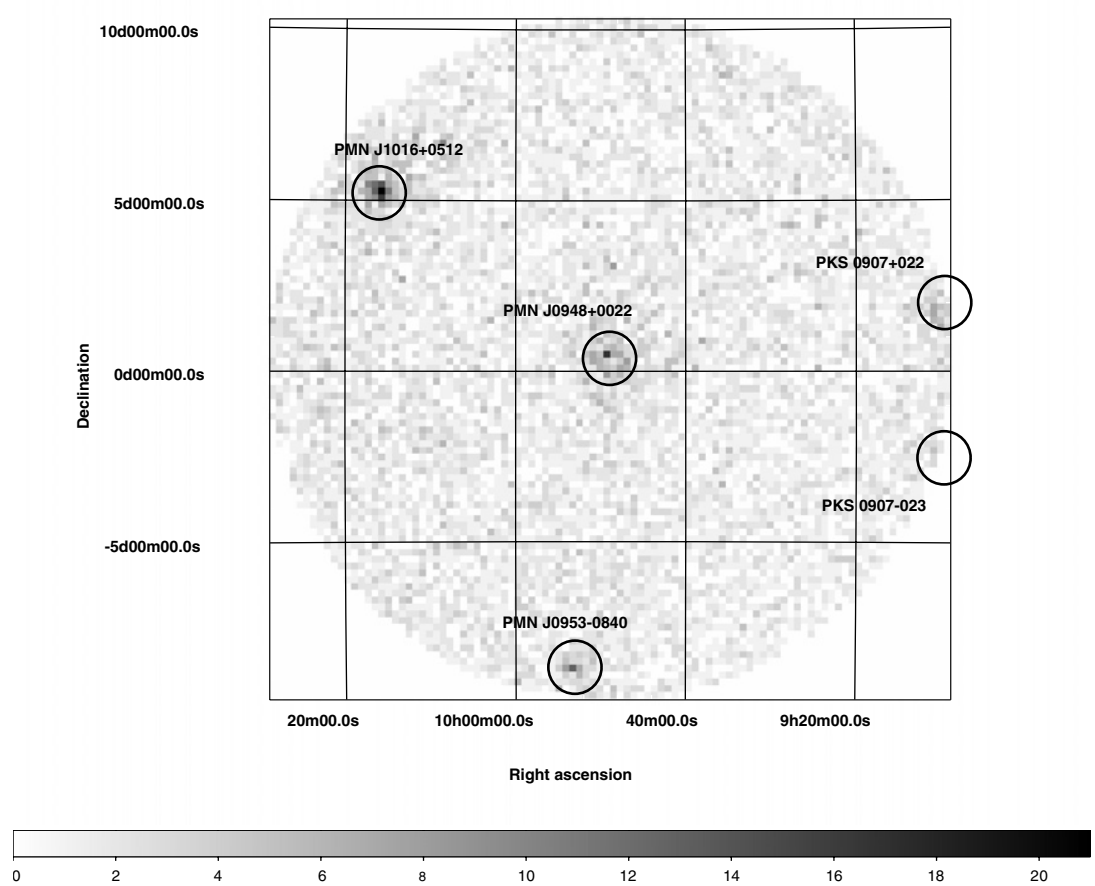

Figure 1. Fermi/LAT counts map ( $E>200 \mathrm{MeV}$ ) of the region centered on PKS J0948+0022 with radius $10^{\circ}$. The pixel size is 0.2 . The gray scale bar is in units of LAT counts integrated in the five-month period. Epoch of coordinates is J2000. Nearby sources included in the likelihood analysis are also indicated.

The data from the LAT (Atwood et al. 2009) were analyzed using the Fermi/LAT Science Tools v 9.8.2, a specific LAT software package. ${ }^{52}$ In summary, we performed the analysis using the guidelines described in detail in the LBAS paper (Abdo et al. 2009b, 2009c), but with some specific features to best fit the characteristics of this source. Events of "Diffuse" class in the Science Tools, coming from zenith angles $<105^{\circ}$ (to avoid Earth's albedo), were extracted from a region with a $10^{\circ}$ radius centered on the coordinates of the radio position of PMN J0948+0022 (R.A. $=09^{\mathrm{h}} 48^{\mathrm{m}} 57^{\mathrm{s}} .6$ and decl. $=+00^{\circ} 22^{\prime} 12^{\prime \prime} .0$, J2000). Because of calibration uncertainties at low energies, data were selected with energies above $200 \mathrm{MeV}$, with no $\gamma$-ray detected at energies $>3 \mathrm{GeV}$. The $\gamma$-ray background is mainly due to three components: the diffuse emission from the Milky Way, the diffuse extragalactic background, and the instrumental background. All these components were modeled and accounted for in the analysis.

An unbinned likelihood algorithm, implemented in the LAT Science Tools as the gtlike task, was used to analyze the data. PMN J0948+0022 was modeled together with four other nearby sources, PKS 0907+022, PKS 0907-023, PMN J1016+0512, and PMN J0953-0840, to take possible contaminations into account, due to the strongly energy-dependent point-spread function (see Figure 1).

The best-fit position of the LAT source, after five months of integration, is R.A. $=09^{\mathrm{h}} 48^{\mathrm{m}} 58^{\mathrm{s}}$ and decl. $=+00^{\circ} 22^{\prime} 48^{\prime \prime}$ (J2000), with a 95\% error radius of $12^{\prime} 36^{\prime \prime}(0.21)$ and detection test statistics $T S=305$ (a $17 \sigma$ detection; see Mattox et al. (1996) for the definition of TS). Figure 1 shows the LAT counts map with the peak of counts consistent with the radio position of PMN J0948+0022. The Figure-of-Merit (FoM) method to associate $\gamma$-ray sources with known radio counterparts was used (Sowards-Emmerd et al. 2003; Abdo et al. 2009b, 2009c). The

\footnotetext{
52 http://fermi.gsfc.nasa.gov/ssc/data/analysis/software/.
}

value of the FoM increased from 18.64 and 93\% confidence level (Bright AGN List, three months of data) to 53.92 and $99 \%$ probability that the association is correct (five months of data).

Figure 2(A) displays the light curve for $E>200 \mathrm{MeV}$ integrated over 10-day time bins between 2008 August 1, 00 UT, and 2009 January 1, 00 UT (MJD 54679 - 54832). The corresponding photon indices $\Gamma$, defined as $F(E) \propto E^{-\Gamma}$, are shown in Figure 2(B). The light curve indicates clear variability of a factor of $\sim 10$ over a timescale of weeks, confirmed with a $\chi^{2}=34.5$ for 14 degrees of freedom (dof) $\left(\tilde{\chi}^{2}=2.5\right)$ for a fit with a constant and an excess variance of $0.19 \pm 0.06$ (see Nandra et al. (1997) for a definition of excess variance). On the other hand, the photon index does not show evident variability and can be fitted with a constant $\left(\tilde{\chi}^{2}=0.80\right)$. The radio data at $15 \mathrm{GHz}$ in Figure 2(C), described in Section 3.3, display no variability during the same time interval. We note, however, that radio data are missing during the episode of variability at $\gamma$ rays at the beginning of the LAT light curve (Figure 2(A)) and a strong radio outburst was observed well before the launch of Fermi.

The spectrum averaged over the whole data set was initially fitted with a single power-law model with $\Gamma=2.6 \pm 0.1$ and flux $(E>200 \mathrm{MeV})$ equal to $(4.0 \pm 0.3) \times 10^{-8} \mathrm{ph} \mathrm{cm}^{-2} \mathrm{~s}^{-1}$ $(T S=298)$. A fit with a broken power-law model gives a slight increase of the TS. The spectral parameters are as follows: the break energy is $E_{\mathrm{b}}=1.0 \pm 0.4 \mathrm{GeV}$, while the photon index for $E<E_{\mathrm{b}}$ is $\Gamma_{1}=2.3 \pm 0.2$ and for $E>E_{\mathrm{b}}$ is $\Gamma_{2}=$ $3.4 \pm 0.5$. The integrated flux $(E>200 \mathrm{MeV})$ is $(3.9 \pm 0.3) \times$ $10^{-8} \mathrm{ph} \mathrm{cm}^{-2} \mathrm{~s}^{-1}(T S=305)$. The likelihood test ratio gives $97 \%$ probability in favor of the broken power-law model with respect to the single power law.

It is worth noting that the quoted errors are statistical only. Systematic errors should be added. According to the studies on the Vela Pulsar (Abdo et al. 2009a), our current conservative estimates of systematic errors are $<30 \%$ for flux measurements 


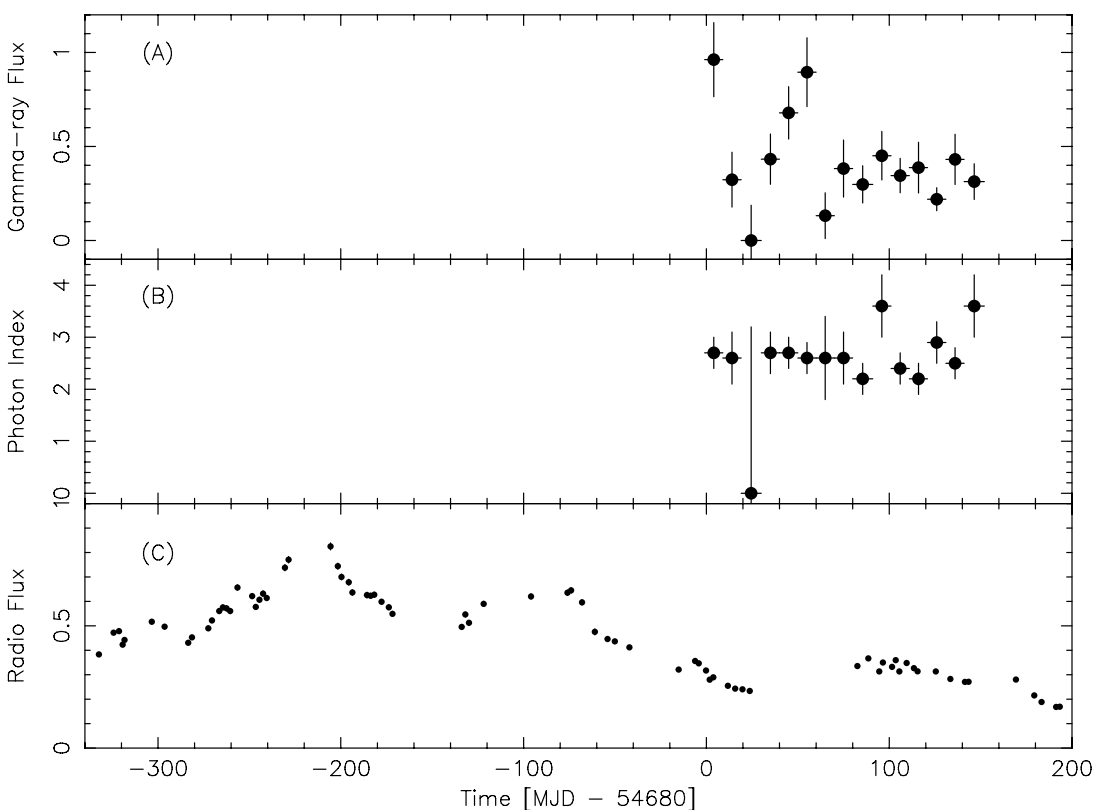

Figure 2. (Panel (A)) Fermi/LAT flux for $E>200 \mathrm{MeV}$ in units of $\left(10^{-7} \mathrm{ph} \mathrm{cm}^{-2} \mathrm{~s}^{-1}\right)$ with 10-day time bins and (panel (B)) the corresponding photon index. (Panel (C)) OVRO radio flux at $15 \mathrm{GHz}(\mathrm{Jy})$. The timescale is from 2007 September 4 at 16:25:41 UTC to 2009 February 11 06:53:48 UTC and the day 0 in the $X$-axis refers to the beginning of LAT observations (2008 August 1).

Table 1

Summary of Results from Analysis of the Swift Data Obtained on 2008 December 5 (ObsID 00031306001 )

\begin{tabular}{|c|c|c|c|c|c|}
\hline \multicolumn{6}{|c|}{ BAT $(20-100 \mathrm{keV})$} \\
\hline & $\begin{array}{l}\text { Exposure } \\
\quad(\mathrm{ks})\end{array}$ & & \multicolumn{3}{|c|}{$\begin{array}{c}\text { Flux } 20-100 \mathrm{keV} \\
\left(10^{-10} \mathrm{erg} \mathrm{cm}^{-2} \mathrm{~s}^{-1}\right)\end{array}$} \\
\hline & 4.3 & & \multicolumn{3}{|c|}{$<7.0$} \\
\hline \multicolumn{6}{|c|}{ XRT $(0.2-10 \mathrm{keV})$} \\
\hline $\begin{array}{l}\begin{array}{l}\text { Exposure } \\
\text { (ks) }\end{array} \\
\end{array}$ & $\begin{array}{c}N_{\mathrm{H}}{ }^{\mathrm{a}} \\
\left(10^{20} \mathrm{~cm}^{-2}\right) \\
\end{array}$ & $\Gamma$ & $\begin{array}{l}\text { Normalization at } 1 \mathrm{keV} \\
\left(10^{-3} \mathrm{ph} \mathrm{cm}^{-2} \mathrm{~s}^{-1} \mathrm{keV}^{-1}\right)\end{array}$ & $\begin{array}{c}\text { Flux }_{2-10 \mathrm{keV}} \\
\left(10^{-12} \mathrm{erg} \mathrm{cm}^{-2} \mathrm{~s}^{-1}\right)\end{array}$ & $\chi^{2} /$ dof \\
\hline 4.2 & 5.22 & $1.83 \pm 0.17$ & $1.5 \pm 0.3$ & $2.2 \pm 0.2$ & $5.1 / 8$ \\
\hline \multicolumn{6}{|c|}{ UVOT (Observed Magnitudes) } \\
\hline $\begin{array}{l}v \\
(5468 \AA)\end{array}$ & $\begin{array}{c}b \\
(4392 \AA)\end{array}$ & $\begin{array}{c}u \\
(3465 \AA)\end{array}$ & $\begin{array}{c}u v w 1 \\
(2600 \AA)\end{array}$ & $\begin{array}{c}u v m 2 \\
(2246 \AA)\end{array}$ & $\begin{array}{c}u v w 2 \\
(1928 \AA)\end{array}$ \\
\hline $18.2 \pm 0.1$ & $18.56 \pm 0.07$ & $17.79 \pm 0.06$ & $17.48 \pm 0.06$ & $17.50 \pm 0.06$ & $17.55 \pm 0.05$ \\
\hline
\end{tabular}

Notes. See the text for details.

${ }^{\text {a }}$ Fixed value from measurements of the Galactic absorption by the Leiden-Argentine-Bonn survey (Kalberla et al. 2005).

and 0.1 for the photon index. Significant reduction of such systematic uncertainties is expected once the calibration of the LAT instrument is completed.

\subsection{Swift}

On 2008 December 5 at 02:25 UTC (MJD 54805.10), Swift observed PMN J0948+0022 (ObsID 00031306001, exposure $\sim 4 \mathrm{ks})$. For the screening, reduction, and analysis of the data from the three instruments (Burst Alert Telescope (BAT), Barthelmy et al. 2005; XRT, Burrows et al. 2005; UVOT, Roming et al. 2005) onboard the Swift satellite (Gehrels et al. 2004), we used the HEASoft v . 6.6.1 software package, together with the CALDB updated on 2008 December 9.

The XRT (0.2-10 keV energy band) was used in the photon counting mode and no evidence of pile-up was found. Data were processed and screened by using the xrtpipeline task with default parameters and grades $0-12$ (the single to quadruple pixel events). The spectrum was rebinned to have at least 30 counts per bin.

No detection was found at $E>10 \mathrm{keV}$ with BAT, after having binned, cleaned from hot pixels, deconvolved, and integrated all the data available in this pointing.

UVOT observed the source with all the six available filters. Data were integrated with the uvotimsum task and then analyzed by using the uvotsource task, with a source region radius of $5^{\prime \prime}$ for the optical filters and $10^{\prime \prime}$ for the UV, while the background was extracted from a circular region $1^{\prime}$-sized and centered in a nearby source-free region. It was not possible to select an annular region centered on PMN J0948+0022 because of nearby sources. The observed magnitudes were dereddened according to the extinction laws of Cardelli et al. (1989) with $A_{V}=0.277$ and then converted into flux densities according to the standard formulae and zero points (Poole et al. 2008).

The results are summarized in Table 1. 


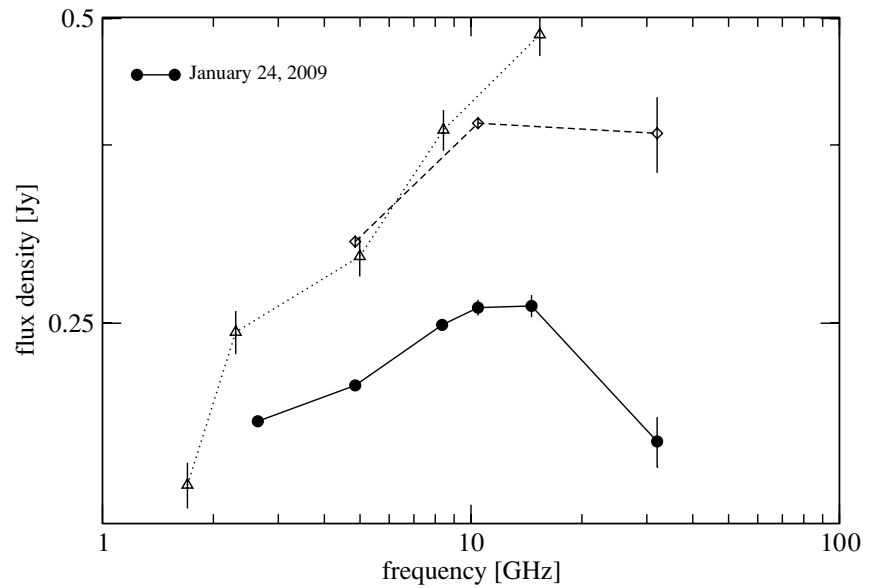

Figure 3. Evolution of the radio spectrum of PMN J0948+022. Filled circles denote the Effelsberg observations of 2009 January. Archival data are shown in gray: triangles represent VLBA measurements conducted in 2003 October (Doi et al. 2006) and diamonds represent archival Effelsberg measurements obtained in 2006 (Vollmer et al. 2008).

\subsection{Radio}

\subsubsection{Effelsberg}

The centimeter spectrum of PMN J0948+0022 was observed with the Effelsberg $100 \mathrm{~m}$ telescope on 2009 January 24 (MJD 54855.5) within the framework of a Fermi-related monitoring program of potential $\gamma$-ray blazars (F-GAMMA project; Fuhrmann et al. 2007). The measurements were conducted with the secondary focus heterodyne receivers at $2.64,4.85,8.35$, $10.45,14.60$, and $32.00 \mathrm{GHz}$. The observations were performed quasi-simultaneously with cross-scans, that is, slewing over the source position, in azimuth and elevation directions, with adaptive numbers of sub-scans for reaching the desired sensitivity (for details, see Fuhrmann et al. 2008; Angelakis et al. 2008). Pointing offset correction, gain correction, atmospheric opacity correction, and sensitivity correction have been applied to the data.

The radio spectrum acquired has a convex shape with a turnover frequency between 10.45 and $14.60 \mathrm{GHz}$ (Figure 3). The low-frequency part spectral index $\alpha_{2.64}^{10.45}$, measured between 2.64 and $10.45 \mathrm{GHz}$, is $-0.18 \pm 0.02$, whereas the highfrequency optically thin spectral index $\alpha_{14.6}^{32}=0.39$.

The comparison of the acquired spectrum with previously observed ones (Vollmer et al. 2008; Doi et al. 2006) reveals that the source is presently in a much lower flux density state (see Figure 3). This indicates intense variability. From the change of the $4.85 \mathrm{GHz}$ flux density over about three years (Vollmer et al. 2008), we estimate a variability brightness temperature (e.g., Fuhrmann et al. 2008) of $1.4 \times 10^{11} \mathrm{~K}$. Assuming the equipartition brightness temperature limit of $\sim 10^{11} \mathrm{~K}$ (Readhead 1994), we obtain a lower limit for the Doppler factor of $\delta>1.4$.

\subsubsection{Owens Valley Radio Observatory}

PMN J0948+0022 has been regularly observed from 2007 September 4 at 16:25 UTC to 2009 February 11 at 06:53 UTC (MJD 54347.68-54873.29) at $15 \mathrm{GHz}$ by the OVRO $40 \mathrm{~m}$ telescope as part of an ongoing Fermi blazar monitoring program of all 1159 Candidate Gamma-Ray Blazar Survey (CGRaBS) blazars north of decl. $-20^{\circ}$ (Healey et al. 2008). Flux densities were measured using azimuth double switching as described in Readhead et al. (1989). The relative uncertainties in flux density

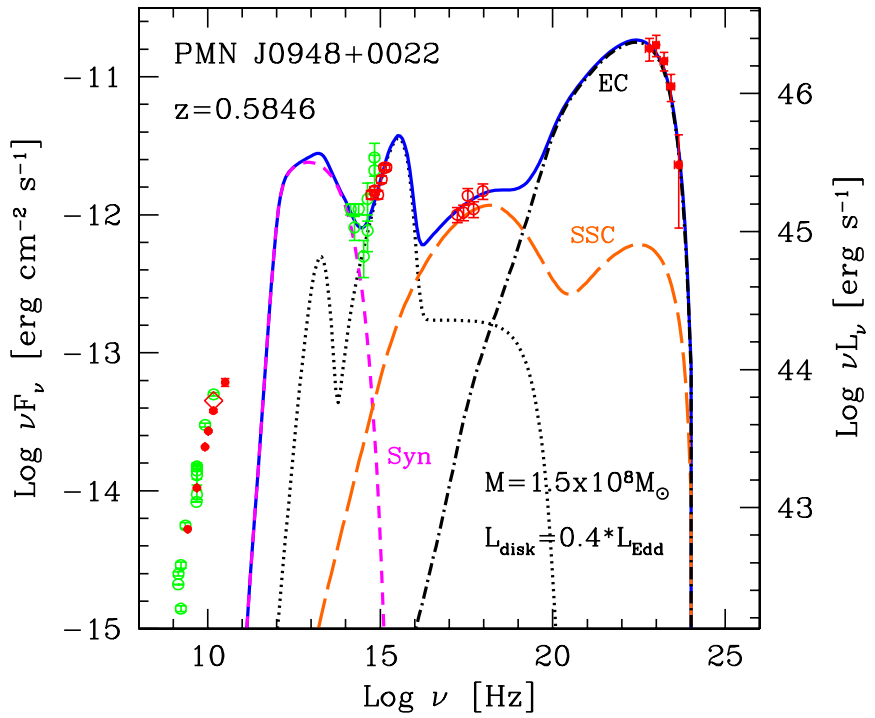

Figure 4. SED of PMN J0948+0022. Fermi/LAT (five months of data), Swift XRT and UVOT (2008 December 5), Effelsberg (2009 January 24) and OVRO (average in the five months of LAT data, indicated with a red diamond) are indicated with red symbols. Archival data are marked with green symbols. Radio data: from 1.4 to $15 \mathrm{GHz}$ from Bennett et al. (1986), Becker et al. (1991), Gregory \& Condon (1991), White \& Becker (1992), Griffith et al. (1995), and Doi et al. (2006). Optical/IR: USNO B1, B, R, I filters (Monet et al. 2003); 2MASS $J, H, K$ filters (Cutri et al. 2003). The dotted line indicates the contributions from the infrared torus, the accretion disk, and the X-ray corona. The synchrotron (self-absorbed) is shown with a small dashed line. The SSC and EC components are displayed with dashed and dot-dashed lines, respectively. The continuous line indicates the sum of all the contributions.

(A color version of this figure is available in the online journal.)

result from a $5 \mathrm{mJy}$ typical thermal uncertainty in quadrature with a $1.6 \%$ systematic uncertainty. The absolute flux density scale is calibrated to about $5 \%$ using the model for $3 \mathrm{C} 286$ by Baars et al. (1977). This absolute uncertainty is not included in the plotted errors.

PMN J0948+0022 has been reported to show variability by a factor of 2 in radio over year timescales (Zhou et al. 2003) and $\sim 31 \%$ fluctuations in month timescales (Doi et al. 2006). The OVRO $40 \mathrm{~m} 15 \mathrm{GHz}$ time series shows a clear structure at timescales down to weeks and year-scale fluctuations by a factor of 4 (Figure 2, panel (C)). The rapid variability we observe in this object — at least $400 \mathrm{mJy}$ in 77 days or $5 \mathrm{mJy} /$ day-enables us to determine a variability brightness temperature of $\sim 2 \times 10^{13}$ $\mathrm{K}$, assuming the $\Lambda \mathrm{CDM}$ cosmology described in Section 1 .

It is often not easy to determine the optically thin spectral index of blazars at radio frequencies because they are complex structures, with different regions becoming optically thin at different radio frequencies. In the present case, the most recent results show a turnover between 10 and $15 \mathrm{GHz}$, and a 15$30 \mathrm{GHz}$ spectral index of $\sim 0.4$, but we do not believe that this is the optically thin spectral index. It is much more likely that one still sees synchrotron self-absorption so that the spectrum between $15 \mathrm{GHz}$ and $30 \mathrm{GHz}$ is much flatter than the true optically thin spectral index. In such cases, it is safer to assume an optically thin spectral index of 0.75 and to use the frequency of observation. These only have a small effect on the derived $T_{\text {eq }}$ unless $\alpha$ is very close to 0.5 , which is too flat, in our view, for an optically thin spectral index in most cases.

The equipartition brightness temperature (Readhead 1994), in the current cosmological model, is then $T_{\text {eq }} \sim 5.5 \times 10^{10} \mathrm{~K}$, assuming an average optically thin spectral index of 0.75 , and hence the equipartition Doppler factor is $\delta \sim 7$, which is typical 
of highly variable blazars. This agrees with Doi et al. (2006), who reported an equipartition Doppler factor $\delta>2.7$, and the Effelsberg lower limit reported in Section 3.3.1. This suggests that, in the compact radio emission regions in this object, the Lorentz $\Gamma$ factor is of order 10.

\section{SPECTRAL ENERGY DISTRIBUTION}

Figure 4 displays the SED built with the Fermi/LAT, Swift, and Effelsberg and OVRO data analyzed in the present work (red symbols) together with archival data (green symbols). Archival radio data are from Bennett et al. (1986), Becker et al. (1991), Gregory \& Condon (1991), White \& Becker (1992), Griffith et al. (1995) and Doi et al. (2006); optical/IR data are from USNO B1 for $B, R, I$ filters (Monet et al. 2003) and from Two Micron All Sky Survey (2MASS) for $J, H, K$ filters (Cutri et al. 2003).

The resulting SED strongly resembles that of a typical high power blazar, with two nonthermal emission peaks in the farIR and between $10^{22}$ and $10^{23} \mathrm{~Hz}(40-400 \mathrm{MeV})$. Also the peak produced by the accretion disk is well defined, due to the UVOT data, extending the photometric coverage to the nearUV. If we assume a standard Shakura \& Sunyaev (1973) disk emission, the UVOT data permit fixing a lower limit to the mass of the black hole, which turns out to be around $10^{8} M_{\odot}$, in agreement with the estimates by Zhou et al. (2003). For lower masses, in fact, the luminosity needed to fit these data becomes super-Eddington.

We model the contemporaneous optical to $\gamma$-ray data with a one-zone synchrotron and IC model, in accordance with the models generally used for blazars. Also for this source, the radio emission is assumed to come from larger-scale emission regions further away along the jet, while the rest of the SED is attributed to the region at the beginning of the jet plus the contribution of the accretion disk. The IR radiation produced by the assumed torus does not influence the derived nonthermal SED, since the corresponding radiation energy density is much smaller than that produced by the lines.

The complete description of the general model used can be found in Ghisellini \& Tavecchio (2009); here, we briefly summarize the main parameters as follows.

1. The source is assumed to be located at a distance $R_{\text {diss }}=$ $6.75 \times 10^{16} \mathrm{~cm}$ from a black hole of mass $M=1.5 \times$ $10^{8} M_{\odot}$.

2. The source is assumed to be a sphere of radius $R=$ $6.75 \times 10^{15} \mathrm{~cm}$ (i.e., a conical jet of semiaperture angle $\psi=0.1 \mathrm{rad}$ is assumed).

3. It moves with a bulk Lorentz factor $\Gamma=10$ at a viewing angle $\theta_{\mathrm{v}}=6^{\circ}$.

4. The disk luminosity is $40 \%$ of the Eddington value $\left(L_{\text {disk }}=\right.$ $\left.9 \times 10^{45} \mathrm{erg} \mathrm{s}^{-1}\right)$.

5. Above (and below) the disk we assume an X-ray emitting corona, producing a luminosity $L_{\text {cor }}=0.3 L_{\text {disk }}$ and with a power-law spectrum (energy index $\alpha_{X}=1$ ), ending in an exponential cut at $150 \mathrm{keV}$.

6. $10 \%$ of the disk emission is assumed to be absorbed and re-emitted by the broad-emission line region (BLR), which emits narrow permitted lines, in this specific case.

7. The BLR is placed at a distance $R_{\mathrm{BLR}}=10^{17} L_{\text {disk, } 45}^{1 / 2}=$ $3 \times 10^{17} \mathrm{~cm}$, where $L_{\text {disk, } 45}$ is the disk luminosity in units of $10^{45} \mathrm{erg} \mathrm{s}^{-1}$.
8. A dusty torus absorbs $10 \%$ of $L_{\text {disk }}$ re-emitting it in the far-IR. Its distance is assumed to be $R_{\mathrm{IR}}=2.5 \times$ $10^{18} L_{\text {disk,45 }}^{1 / 2} \mathrm{~cm}$, calculated by considering that the dust is at a temperature of $370 \mathrm{~K}$ (see Cleary et al. 2007), which is the maximum of efficiency in reprocessing the impinging photons into IR radiation.

For the above choice of parameters, the BLR is the main producer of the seed photons for the IC scattering in the emitting region. In Figure 4, this component is labeled EC. On the other hand, the SSC radiation is important in the X-ray band, where it dominates the radiative output (the two-peak shape of the SSC curve corresponds to the first- and second-order scattering). In this scenario, that is, with the X-ray data due to the SSC emission, the magnetic field $B$ is strongly constrained, since it controls the value of the SSC emission. In our case, $B=3.2 \mathrm{G}$.

The particle energy distribution of the emitting electrons is calculated by the continuity equation, assuming that the injected electrons are distributed in energy according to a smoothly joining broken power law of slopes $\gamma^{-1}$ and $\gamma^{-2.2}$ below and above $\gamma=800$, respectively. The maximum electron energy corresponds to a random Lorentz factor of $\gamma_{\max }=1600$ and the IC peak corresponds to $\gamma_{\text {peak }}=411$. The total power injected in the form of relativistic electrons is $L_{\mathrm{e}}^{\prime}=2.4 \times 10^{43} \mathrm{erg} \mathrm{s}^{-1}$, as calculated in the comoving frame.

Once accelerated, the bulk kinetic power carried by the electrons in the jet is $L_{\mathrm{e}}=5.0 \times 10^{44} \mathrm{erg} \mathrm{s}^{-1}$, to be compared with the Poynting flux $L_{\mathrm{B}}=1.8 \times 10^{44} \mathrm{erg} \mathrm{s}^{-1}$ and the power in radiation $L_{\mathrm{rad}}=2.0 \times 10^{45} \mathrm{erg} \mathrm{s}^{-1}$. As occurs in typical powerful blazars (see, e.g., Celotti \& Ghisellini 2008, also for the exact definitions of these powers), the radiation observed carries more power than what is available in the magnetic field and electron energy. Thus, we require that the jet also carries protons: if we assume one proton per electron, we obtain $L_{\mathrm{p}}=4.8 \times 10^{46}$ $\mathrm{erg} \mathrm{s}^{-1}$. This value can be reduced by assuming the presence of pairs.

The impact of the presence of narrow permitted lines in the BLR can be evaluated in two ways. First, there can be a geometrical explanation: if the BLR is torus-like and we are observing it face-on, then the Doppler broadening is reduced and the line width is smaller than usual. A similar geometry has been invoked by Decarli et al. (2008) to explain why NLS1 do not have small masses.

This BLR geometry, although different from what is used in the adopted model, does not affect any of the model results, since the important parameter is the angle at which the jet sees the BLR. Second, it is still possible to consider an isotropic geometry of the BLR and take into account the effect of the radiation pressure, as proposed by Marconi et al. (2008). The BLR is then moved farther off the central singularity $\left(R_{\mathrm{BLR}}=5 \times 10^{17} \mathrm{~cm}\right)$, but the impact in the model parameters mainly results in an increase of $\Gamma$ from 10 to 13 . Other new values of parameters are $\gamma_{\max }=1500, \gamma_{\text {peak }}=430 L_{\mathrm{e}}^{\prime}=2.6 \times$ $10^{43} \mathrm{erg} \mathrm{s}^{-1}, B=2.01 \mathrm{G}$. The power output of the jet is $L_{\mathrm{e}}=1.2 \times 10^{45} \mathrm{erg} \mathrm{s}^{-1}, L_{\mathrm{B}}=1.3 \times 10^{44} \mathrm{erg} \mathrm{s}^{-1}$, $L_{\mathrm{rad}}=3.2 \times 10^{45} \mathrm{erg} \mathrm{s}^{-1}$, and $L_{\mathrm{p}}=7.9 \times 10^{46} \mathrm{erg} \mathrm{s}^{-1}$.

It is worth noting that the systematics in the LAT data do not affect significantly the results of the modeling. Indeed, in the case of a LAT flux equal to $130 \%$ of the observed value (the worst case), it is sufficient to increase a little the injected power $L_{\mathrm{e}}^{\prime}$ (from $2.4 \times 10^{43}$ to $2.8 \times 10^{43} \mathrm{erg} \mathrm{s}^{-1}$ ) and to decrease a little the magnetic field $B$ (from 3.2 to $2.5 \mathrm{G}$ ). The derived 

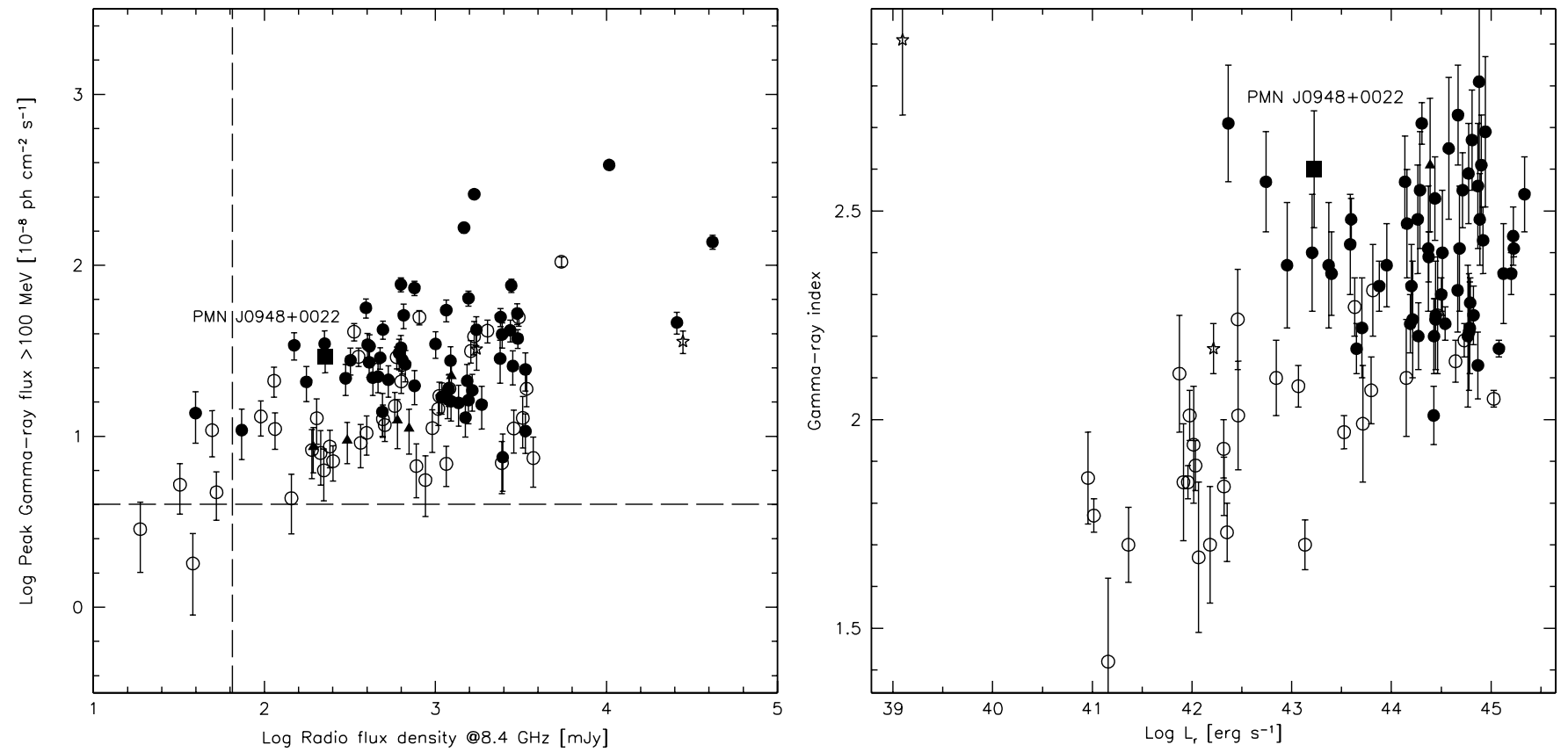

Figure 5. Radio vs. $\gamma$-ray properties of PMN J0948+0022 (indicated with a filled square) compared with the other blazars detected by LAT (figure adapted from Figure 14 in Abdo et al. 2009c). (Left panel) Peak $\gamma$-ray flux $(E>100 \mathrm{MeV})$ vs. radio flux density at $8.4 \mathrm{GHz}$; the dashed lines show the CRATES flux density limit and the typical LAT detection threshold. (Right panel) $\gamma$-ray photon index vs. radio luminosity.

quantities become $L_{\mathrm{e}}=6.3 \times 10^{44} \mathrm{erg} \mathrm{s}^{-1}, L_{\mathrm{B}}=1.0 \times$ $10^{44} \mathrm{erg} \mathrm{s}^{-1}, L_{\mathrm{rad}}=2.5 \times 10^{45} \mathrm{erg} \mathrm{s}^{-1}$, and $L_{\mathrm{p}}=5.0 \times 10^{46} \mathrm{erg}$ $\mathrm{s}^{-1}$. A change in the $\gamma$-ray photon index equal to \pm 0.1 results in very negligible changes in the SED.

The model parameters used to explain the SED of PMN J0948+0022 are rather well constrained, given the basic assumptions of the model. However, they may not be unique. Another solution might be possible if we assume that the dusty torus contributing to the IR seed photons is hotter and more compact than assumed here. It must be hotter because otherwise we need too energetic electrons to fit the hard X-ray emission (by EC), and these very same electrons would overproduce (by synchrotron) the observed emission in the optical. A stratified and clumpy torus (e.g., Nenkova et al. 2008) could work. Also in this "hot and clumpy torus" case we can obtain a reasonable representation of the data (i.e., the same ratios between the radiation energy densities - by both synchrotron and external photons-and the magnetic energy density), at distances one order of magnitude larger than assumed here. As a consequence, the emitting region should be one order of magnitude larger, and thus should vary on longer timescales. The detection of a typical variability timescale can therefore discriminate between the two possible solutions.

\section{DISCUSSION AND CONCLUSIONS}

Our findings clearly show that PMN J0948+0022, the first narrow-line quasar detected in $\gamma$-rays, hosts a relativistic jet with the SED very similar to those of "classical" FSRQs.

The parameters relevant for the spectral shape of the SED derived from the modeling, using the approach by Ghisellini \& Tavecchio (2009), are consistent with those of high-power blazars, though at the lower limit in power of the FSRQ region as derived, for example, in Celotti \& Ghisellini (2008) for a large sample of blazars (cf. their Figure 2 and Figure 6). The comparison of radio versus $\gamma$-ray properties shows that in both bands PMN J0948+0022 has a relatively low power with respect to the other FSRQ detected by LAT to date, although no striking differences appear (Figure 5). Effelsberg and OVRO radio observations also show characteristics similar to those of FSRQs.

We believe that PMN J0948+0022 could be one of the first examples of a FSRQ with a relatively small mass but high accretion rate in terms of the Eddington ratio. This would fit well with the scenario in which the transition between highpeaked and low-peaked blazars is not related to absolute power but to the Eddington ratio as indicated by the FRI-FRII separation which depends on the mass. It remains to be seen whether the jets in other radio-loud NLS1 conform to this scenario. Fermi is expected to answer this question by measuring the $\gamma$-ray luminosity and spectra of the most radio-loud ones.

The Fermi/LAT Collaboration acknowledges generous ongoing support from a number of agencies and institutes that have supported both the development and the operation of the LAT as well as scientific data analysis. These include the National Aeronautics and Space Administration and the Department of Energy in the United States, the Commissariat à l'Energie Atomique and the Centre National de la Recherche Scientifique/Institut National de Physique Nucléaire et de Physique des Particules in France, the Agenzia Spaziale Italiana and the Istituto Nazionale di Fisica Nucleare in Italy, the Ministry of Education, Culture, Sports, Science and Technology (MEXT), High Energy Accelerator Research Organization (KEK), and Japan Aerospace Exploration Agency (JAXA) in Japan, and the K. A. Wallenberg Foundation, the Swedish Research Council, and the Swedish National Space Board in Sweden.

Additional support for science analysis during the operations phase from the following agencies is also gratefully acknowledged: the Istituto Nazionale di Astrofisica in Italy and the K. A. Wallenberg Foundation in Sweden for providing a grant in support of a Royal Swedish Academy of Sciences Research fellowship for J.C. 
This research is partly based on observations with the $100 \mathrm{~m}$ telescope of the Max-Planck-Institut für Radioastronomie (MPIfR) at Effelsberg. The monitoring program at the OVRO is supported by NASA award NNX08AW31G and NSF award AST-0808050.

This research has made use of the NASA/IPAC Extragalactic Database (NED) which is operated by the Jet Propulsion Laboratory, California Institute of Technology, under contract with the National Aeronautics and Space Administration. This research has made use of data obtained from the High Energy Astrophysics Science Archive Research Center (HEASARC), provided by NASA's Goddard Space Flight Center.

\section{REFERENCES}

Abdo, A. A., et al. 2009a, ApJ, 696, 1084

Abdo, A. A., et al. 2009b, ApJ, in press (arXiv:0902.1340)

Abdo, A. A., et al. 2009c, ApJ, in press (arXiv:0902.1559)

Aharonian, F., et al. 2008, A\&A, 478, 387

Angelakis, E., Fuhrmann, L., Marchili, N., Krichbaum, T. P., \& Zensus, J. A. 2008, Mem. SAIt, 79, 1042

Atwood, W. B., et al. 2009, ApJ, 697, 1071

Baars, J. W. M., Genzel, R., Pauliny-Toth, I. I. K., \& Witzel, A. 1977, A\&A, 61,99

Barthelmy, S. D., et al. 2005, Space Sci. Rev., 120, 143

Becker, R. H., White, R. L., \& Edwards, A. L. 1991, ApJS, 75, 1

Bennett, C. L., Lawrence, C. R., Burke, B. F., Hewitt, J. N., \& Mahoney, J. 1986, ApJS, 61, 1

Blandford, R., \& Rees, M. 1978, in Pittsburgh Conference on BL Lac Objects, ed. A. N. Wolfe (Pittsburgh, PA: Pittsburgh Univ. Press), 328

Blandford, R. D., \& Levinson, A. 1995, ApJ, 441, 79

Błażejowski, M., Sikora, M., Moderski, R., \& Madejski, G. M. 2000, ApJ, 545, 107

Boroson, T. A. 2002, ApJ, 565, 78

Böttcher, M., \& Dermer, C. D. 2002, ApJ, 564, 86

Burrows, D. N., et al. 2005, Space Sci. Rev., 120, 165

Cardelli, J. A., Clayton, G. C., \& Mathis, J. S. 1989, ApJ, 345, 245

Cavaliere, A., \& D'Elia, V. 2002, ApJ, 571, 226

Celotti, A., \& Ghisellini, G. 2008, MNRAS, 385, 283

Cleary, K., et al. 2007, ApJ, 660, 117

Cutri, R. M., et al. 2003, The IRSA 2MASS All-Sky Point Source Catalog NASA/IPAC Infrared Science Archive

Decarli, R., Dotti, M., Fontana, M., \& Haardt, F. 2008, MNRAS, 386, L15

Dermer, C. D., Schlickeiser, R., \& Mastichiadis, A. 1992, A\&A, 256, L27

Doi, A., et al. 2006, PASJ, 58, 829

Falcone, A. D., et al. 2004, ApJ, 613, 710

Fender, R., \& Belloni, T. 2004, ARA\&A, 42, 317

Foschini, L., et al. 2009, Adv. Space Res., 43, 889

Fossati, G., Maraschi, L., Celotti, A., Comastri, A., \& Ghisellini, G. 1998, MNRAS, 299, 433
Fuhrmann, L., Zensus, J. A., Krichbaum, T. P., Angelakis, E., \& Readhead, A. C. S. 2007, in AIP Conf. Proc. 921, The First GLAST Symposium, ed. S. Ritz, P. Michelson, \& C. A. Meegan (Melville, NY: AIP), 249

Fuhrmann, L., et al. 2008, A\&A, 490, 1019

Gehrels, N., et al. 2004, ApJ, 611, 1005

Ghisellini, G., \& Celotti, A. 2001, A\&A, 379, L1

Ghisellini, G., Celotti, A., Fossati, G., Maraschi, L., \& Comastri, A. 1998, MNRAS, 301, 451

Ghisellini, G., Maraschi, L., \& Treves, A. 1985, A\&A, 146, 204

Ghisellini, G., \& Tavecchio, F. 2008, MNRAS, 387, 1669

Ghisellini, G., \& Tavecchio, F. 2009, MNRAS, in press (arXiv:0902.0793)

Gregory, P. C., \& Condon, J. J. 1991, ApJS, 75, 1011

Griffith, M. R., Wright, A. E., Burke, B. F., \& Ekers, R. D. 1995, ApJS, 97, 347

Healey, S. E., et al. 2008, ApJS, 175, 97

Kalberla, P. M. W., et al. 2005, A\&A, 440, 775

Komatsu, E., et al. 2009, ApJS, 180, 330

Komossa, S., et al. 2006, ApJ, 132, 531

Maraschi, L. 2001, in AIP Conf. Proc. 586, 20th Texas Symposium on Relativistic Astrophysics, ed. J. C. Wheeler \& H. Martel (New York: AIP), 409

Maraschi, L., Foschini, L., Ghisellini, G., Tavecchio, F., \& Sambruna, R. M. 2008, MNRAS, 391, 1981

Maraschi, L., \& Tavecchio, F. 2001, in ASP Conf. Ser. 227, Blazar Demographics and Physics, ed. P. Padovani \& C. Megan Urry (San Francisco, CA: ASP), 40

Maraschi, L., \& Tavecchio, F. 2003, ApJ, 593, 667

Marconi, A., et al. 2008, ApJ, 678, 693

Mattox, J. R., et al. 1996, ApJ, 461, 396

Monet, D. G., et al. 2003, AJ, 125, 984

Nandra, K., George, I. M., Mushotzky, R. F., Turner, T. J., \& Yaqoob, T. 1997, ApJ, 476, 70

Nenkova, M., Sirocky, M., Ivezić, Z., \& Elitzur, M. 2008, ApJ, 685, 147

Padovani, P. 2007, Astrophys. Space Sci., 309, 63

Pogge, R. W. 2000, New Astron. Rev., 44, 381

Poole, T. S., et al. 2008, MNRAS, 383, 627

Readhead, A. C. S. 1994, ApJ, 426, 51

Readhead, A. C. S., et al. 1989, ApJ, 346, 566

Rees, M. J. 1966, Nature, 211, 468

Rodríguez-Ardila, A., Binette, L., Pastoriza, M. G., \& Donzelli, C. J. 2000, ApJ, 538,581

Roming, P. W. A., et al. 2005, Space Sci. Rev., 120, 95

Shakura, N. I., \& Sunyaev, R. A. 1973, A\&A, 24, 337

Sikora, M., Begelman, M. C., \& Rees, M. J. 1994, ApJ, 421, 153

Sowards-Emmerd, D., Romani, R. W., \& Michelson, P. F. 2003, ApJ, 590, 109

Urry, C. M., \& Padovani, P. 1995, PASP, 107, 803

Vollmer, B., Krichbaum, T. P., Angelakis, E., \& Kovalev, Y. Y. 2008, A\&A, 489, 49

White, R. L., \& Becker, R. H. 1992, ApJS, 79, 331

Yuan, W., et al. 2008, ApJ, 685, 801

Zhou, H.-Y., et al. 2003, ApJ, 584, 147

Zhou, H.-Y., et al. 2006, ApJS, 166, 128 